\title{
45 years of "Baldin autumn"
}

\author{
Nelly Konopleva* \\ †"VNIIEM" Corporation" JSC,Moscow, Russia \\ E-mail:nellyatheor.jinr.ru
}

In the autumn of 1969 International Seminar "Vector mesons and electromagnetic interactions" took place in Dubna. It was organized by academician M.A.Markov and prof. A.M.Baldin. The ideas and experimental results submited for consideration of this Seminar contained many new approaches in theoretical and experimental physics of elementary particles. In particular new quantization method of the gauge fields by Feynman path integration was proposed by L.D.Faddeev, and new geometric interpretation of the gauge fields in terms of fibre bundle geometry was proposed by N.P.Konopleva. The results, which were under discussion at the Seminar, helped to overcome the deep crisis in elementary particle physics of the 60th. This crisis was induced by following facts: 1) a quantity of new elementary particles was discovered at that time; 2) it was found that some of them are non-elementary (non-point) and have internal structure; 3) in the high energy region nuclear forces influence on electromagnetic processes. At the same time it was absent suitable method of elementary particle classification. It was necessary to construct new models of proton, neutron, and other particles. It was shown that quantum electrodynamics is insufficient for description of new phenomenological results. Any consequent theory of weak and strong nuclear forces was not exist. All these questions were considered at the autumn 1969 Seminar in Dubna. This Seminar generated set of Baldin Seminars on high energy physics problems named "Baldin autumn". They are dedicated to relativistic nuclear physics and quantum chromodynamics problems.

XXII International Baldin Seminar on High Energy Physics Problems

15-20 September, 2014

JINR, Dubna, Russia

\footnotetext{
* Speaker.

${ }^{\dagger}$ It is a pleasure to thank the Organizers of the International Seminar XXII for their support of this investigation
} 


\section{Introduction}

In the 60th the main problem of elementary particle physics was too many particles, which were experimentally discovered at that time. Relativistic invariance was insufficient to classify them and understand the character of their interactions. Internal symmetries were introduced to describe the experimental results. But the origin and physical sense of internal symmetries remained obscure.

Quantum electrodynamics was only fundamental physical theory which could describe interactions of elementary particles. Its predicts of some physical experiment results were surprisingly exact. But experimentalists made use of phenomenological models as a basis.

Important properties of electrodynamics are its linearity, relativistic invariance, and gradient invariance (global and local). Just the last invariance turned later on into gauge invariance. Global invariance means that transformation parameters of the symmetry group are independent of spacetime point. Local invariance means that transformation parameters of symmetry group depend on space-time point.

Invariance of any physical theory with respect to some symmetry group generates the conservation laws. In the frame of Lagrangian formalism the symmetry of theory with respect to Lie group lead to appearance of conservative value set which characterize objects being described by this theory. It is possible two variants.

When the Lie group is a global symmetry, the conservation laws can be construct by the first Noether theorem. They have the form of null divergences of the currents. Integrals of these divergences become the motion integrals. Impuls, energy, moment, spin are just such integrals. They appear in relativistic invariant theories. In quantum theory they are considered as quantum numbers of the objects. Under interactions elementary particles exchange their quantum numbers. The conservation laws, being generated by the global symmetry, are realized on the motion equation solutions only (i.e. are the corollary of them).

Global gradient invariance of electrodynamics generates the conservation law of the electric charge. But why is the local gradient invariance necessary?

When the Lie group is a local symmetry, the conservation laws can be construct by the second Noether theorem. They have the form of covariant conservation laws and involve other variable. Usual conservation laws corresponding with the first Noether theorem are not canceled, but they become the identities on the motion equation solutions. The conservation laws, being generated by the local symmetries, are nontrivial outside of the motion equation solutions. Such conservation laws are in Einsteinian gravitation theory. In the Maxwell electrodynamics the local gradient invariance does not cancel the conservation law of electrical charge. This law is fulfiled on the solutions of the field equations automatically. But outside of them it pass into the conservation law of charge density. Difference between these two laws is not appreciable because of electrodynamics linearity. So, in Maxwell electrodynamics due to the local gradient invariance it is kept not only electrical charge, but its density also.

After Hofstadter's experiments which showed that nucleons are not points, but have internal structure (50th of the XX century), elementary particle model building became very popular. It was found that elementary particles are not elementary! Electromagnetic interactions in region of high energies pointed to deviation from quantum electrodynamics predictions. 


\section{Where is the limit of electrodynamics?}

Classical elektrodynamics is fundamental theory, which is proved very carefully. It describes long-range forces, and its predicts are veryfied from microworld to the distances of Universe scale. Electromagnetic field quantum - photon - interacts universally with all charged elementary particles. In classical and quantum electrodynamics electrons are regarded as a point particles. Discovery of intrinsic proton structure put the question about the same properties of electrons. But until now electron behavior is corresponding with behavior of rigid point ball.

In 1961 American physicist Hofstadter was awarded the Nobel Prize in Physics for his high energy nucleon-electron scattering experiments, which nucleon structure was found in. He showed not only that proton and neutron are not point particles, but they are in essence the same object with isospin third component differing them from each other.

In Hofstadter's experiments the elastic scattering cross-section, being experimentally measured, was compared with that calculated by quantum electrodynamics and Dirac theory. Deviation of theoretical and experimental results demanded an explanation from scientists. Nambu assumed that inside nucleons are new heavy mesons $(\omega)$, which were soon found. Sakurai, Frezer and others introduced also new heavy mesons for explanation of new experimental results. Many new resonanses appeared in 60th. In 1968 L.W.Alwarez was awarded the Nobel Prize for descovery of resonanses in quantities. In 1961 - 1963 vector $\rho$ - and $\phi$ - mesons were also discovered. Theorists recommended, experimenters found. It was beginning of intensive model building. Classification of particle collection fulfiled by symmetry groups. In particularly, $S U(2)$ and $S U(3)$ groups were most succesful. In 1963 J.Vigner was awarded the Nobel Prize in Physics for his contribution to theory of the atomic nucleus and the elementary particles, particularly through the discovery and application of fundamental symmetry principles. In 1969 M.Gell-Mann got the Nobel Prize for his contributions and discoveries concerninmg the classification of elementary particles and their interactions. His famous "the eightfold way" and quark models of elementary particles, proposed by him in 1961, begun their triumphal procession in theoretical physics. But in spite of this success "the eightfold way" remained abstract phenomenological scheme without any reasons. Gauge group of this theory is global $S U(3)$.

Simultaneously many nonlocal model of elementary particles and their interactions were proposed, in particularly, semiclassical one, taking into account the possibility of elementary length existence. Some of them assumed that into the region of elementary length radius the structure of space-time geometry can differ from Minkowski geometry (Markov, Kadyshevski and others).

Hofstadter's experiments showed that at small distances nuclear forces begin to influence on electromagnetic processes. But what way is it happening?

Some of scientists proposed that in this case elastic scattering is the result of exchange by new intermediate vector meson. Then it must be formulated the corresponding theory of such interactions. Simplest model of such sort was the vector dominance model. Later it was replaced by the gauge field theory.

\section{Gauge field theory}

In the 50th Yang, Mills and Utiyama proposed the new approach containing classical nonlin- 
ear field equations for description of nonelectromagnetic interactions of elementary particles. The structure of these equations was similar to the Maxwell equation. But nonlinearity of them made impossible their quantization by usual methods. In 1963 Feynman showed that in this case his formulation of quantum electrodynamics, proposed in 1949, is directly inapplicable also. But in 1965 R.Feynman (together with S.I.Tomonaga and J.Schwinger) was awarded the Nobel Prize in Physics for their fundamental works in quantum electrodynamics, with deep ploughing consequences for the physics of elementary particles.

Yang and Mills (1954) wanted for the theory of weak interactions of elementary particles. In this theory "weak charge" $g$ is introduced instead of electrical charge $e$. Instead of electrodynamical 1-dimentional (abelian) gauge group in Yang-Mills theory $S U(2)$ - non-Abelian gauge group was being use. Nonlinearity of Yang-Mills equations is the corollary of the fact that the gauge group of this theory is non-abelian. Moreover, in contrast to massless photon, which is interaction carrier in electrodynamics, vector bosons of Yang-Mills theory must be very massive because short-range weak interaction. Therefore Yang-Mills theory was non-renormalizable. It means that under quantization its predictions, being obtained by perturbation theory, become senseless. All these problems induced strong scepticism between theoretists. But on the other hand fundamental ideas of universality and symmetry principles, lying in the base of Yang-Mills theory, were too attractive.

Situation was changed when unified model of electromagnetic and weak interaction was proposed independently by S.Weinberg and A.Salam (1967). In this model electromagnetic and weak forces were regarded as the forces in essence of united nature. In addition Weinberg-Salam model included one more gauge field, which described the interactions of elementary particles carried hypercharge. The gauge field of such kind Sakurai proposed to introduce yet in 1960 .

Utiyama (1956) proposed to describe all known interactions as universal vector interactions, which theories differ from each other only by gauge symmetry group. Thus the interaction classification appeared. In its frame nuclear forces were described by $S U(3)$ gauge group, which is non-Abelian similar to $S U(2)$. The charge in the gauge theory of nuclear interactions was, in correspondence with Gell-Mann-Zweig and Bogoliubov-Tavkhelidze proposals, named "color" (1964-1965), and the theory was named chromodynamics. After elimination of above-mentioned problems, which are peculia to all gauge field theories with non-Abelian gauge symmetry group, chromodynamics together with Yang-Mills theory and electrodynamics became a part of Standard Model of elementary particle interactions. New experimental data confirmed correctness of this model.

Improvement of the Feynman method (the method of path integration) was proposed in 1967 by L.D.Faddeev and V.N.Popov. At the same time they, as well as De Witt, quantized YangMills fields. Development of this approach, combined with renormalization procedure realized for massless Yang-Mills fields Taylor (1970) and Slavnov(1971), and for massive Yang-Mills fields 't Hooft (1971), permitted to produce correct calculations by perturbation theory.

But one cloud remaines in that way: gravity is beyond the Standard Model. How could gravity be joined with other interactions?

Here is necessary to differ two view-points on this problem. Because of gravity is clousely connected with the space-time structure, the theory of this interaction is clousely connected with the ways of space-geometry construction. Two basic methods are usually being used: 1) it is 
postulated that space-time geometry is flat, and gravity is usual interaction on this background;2) it is postulated that gravity interaction determines space-time geometry.

Newtonian theory of gravity is constructed by the first postulate. Einsteinian theory of gravity is based on the second postulate. The elementary particle theory is constructed as the particle theory in flat space-time. Its formulation extends Newtonian theory. Relativistic theory of gravity in the flat space-time was developed by V.Fock and A.Logunov with their collaborators. In order to construct the elementary particle theory similar to General Relativity it is necessary to clarify meaning of the gauge fields as geometrical objects. My works were dedicated to this problem.

It is exists the third way of unification gravity and other interactions. In this case all symmetries are regarded as abstract degrees of freedom independently from their origin. Internal and spacetime symmetries are joined in unique group of supersymmetry. This approach is generalization of phenomenological "the eightfold way". It is assumed that the particles of spin 2 describe gravity.

\section{Gravity and gauge fields}

It can be shown that on classical level unified theory of all fundamental interactions including Einsteinian gravity exists. It is the geometric theory of the gauge fields. In it Einsteinian General Relativity is considered the specific case of the gauge theory, when the gauge field is given by the symmetrical tensor of rank two. The question is: what is graviton from geometric point of view?

In General Relativity gravity is closely connected to space-time structure. Therefore the physics objects (gravitons) can be regarded as geometric one.

Riemann was the first who decided to connect geometry of space with the properties of the matter filling it (in 1854, published in 1868). He constructed new geometry step by step. Infinitesimal interval was only given in neighbourhood of every point. Such a geometry reflected the well known fact consisting in that all our experiments are local one. If the matter distribution is continuous it is necessary to find the way connecting the points with each other. The connection coefficients in Riemannian space were input by Christoffel in 1869. But only in 1915 when Hilbert and Einstein proposed their equations it became possible to study the properties of Riemannian spaces as a whole. In this field of knowledge very important results were obtained by A.Z.Petrov in 50-60th of XX century. He constructed two kinds of classifications Einsteinian spaces: algebraic (local one) and in accordance with their motion groups (global one). Lorentz symmetry of usual space-time is one of motion group symmetries.

In 1868 geometry of Riemannian kind was also proposed by Helmholtz, who was the teacher of M.Planck, and M.Planck was a teacher of A.Einstein. Helmholtz was also the first who established in 1847 the meaning of the energy conservation principle in different fields of knowledge.

In 1872 F.Klein proposed to describe the geometric properties of figures placed into some space by means of the invariants of motion groups of this space. In this case the space as a whole must be a symmetrical one. Its symmetry properties describe by means of the Lie groups, which transformations have finite number of parameters.

Evidently Kleinian geometry point of view sharply differ from Riemannian that. As A.Z.Petrov showed, Riemannian space can have not any symmetry in Kleinian sense. Klein strived to connect geometry with properties of the measuring procedures, but not the matter filling the space. 
In spite of the seeming contradiction, both above-mentioned approachs found their applications in physics. Kleinian approach works in Special Relativity and elementary particle physics. Riemannian approach is being used in General Relativity and Gauge Field Theory. Moreover Gauge Field Theory exists in two forms: geometric one (similar to GR) and form of fields in a flat space-time (similar to SR). It became possible due to extension of Einsteinian equivalence principle, which connected Riemannian geometry structure with the local measuring procedures. E.Cartan could geometrically realize these ideas and unify Riemannian and Kleinian points of view (Riemannian geometry in orthogonal frame of reference).

Geometric structure of space can being generated by nongravitational fields, when it is assumed that measurers are placed in different points of space and parameters of their measurements can change from point to point. In this case the gauge field vector-potential play the role of connection coefficients of fiber bundle. The role of such space fiber will play the range of parameter variations. The base of the fiber bundle space must be chousen Riemannian space-time. In this construction equivalence principle extends at all nongeometrical measurements. All internal and space-time symmetries become the local one.

Symmetry group localization leads to modification of the conservation law form. In particular, in consequence of translation group localization the energy-momentum conservation law becomes covariant one. In the frame of Lagrangian formalism for infinite Lie groups, which was worked out by author in 1967, this conservation law is Noether identities corresponding with her second theorem. In the case of GR Noether identities are generated by generally covariant transformations of the space-time coordinates, which are considered the local translations. In the gauge field theory the problem of the gravity energy-momentum pseudotensor is not exist. Appearance of the pseudotensor kind structure points to localization of the symmetry group of the present theory. This phenomenon characterizes the form modifications of all conservation law. New form of the conservation laws (covariant conservation laws) is given by Noether identities of her second theorem for the every concrete local symmetry. Precisely these identities permit to treat geometrically the interactions of elementary particles and show which way it must be done in. The dynamical constants are obtained by integration of the corresponding differential covariant conservation laws. This question is important in connection with the energy problem in the elementary particle theory, astrophysics and cosmology. These results were obtained in 1967 by Lagrangian formalism for infinite Lie groups, which I worked out then. For integration of tensor energy-momentum conservation law arising in GR the new procedure was found. It makes use of Gauss theorem only partially. For this procedure the Riemannian space properties as a whole was necessary. Petrov's classification of Einsteinian spaces with respect to their motion groups made possible mathematically strict displacement of dynamical constant definition from flat spaces to Riemannian one. By Petrov's classification application it was shown that motion constants in Riemannian space-time are the vectors in the Lie algebra of the motion group of corresponding Riemannian space-time. Therefore the number of the motion constants coincides with the number of such vectors.

It is need to note that the important peculiarity of the motion in the Einsteinian space-time is following:

- In absence of the cosmological term relativistic invariance of the theory (10 the motion constants) is only possible when Einsteinian equations describe vacuum space-time, i.e. in ab- 
sence of the particles.

- In presece of the cosmological term 10-parameters motion group is possible, but it is not Poincaré group. It will be De Sitter symmetry group. In that space-time the collection of the motion integrals will be differ from that of relativistic invariant theory in flat space-time.

In De Sitter space rectilinear motions do not exist, because De Sitter group is the group of 5D-rotations. It has not any invariant subgroup which could be analogous to the group of 4D translations being subgroup of Poincaré group. Therefore in de Sitter space the components of 4-momentum are not individually conservative. Consequently individually invariant 3momentum components and energy do not exist. In spite of total number of dynamical constants in de Sitter and Minkowski spaces are the same, these constants have different nature. Absence of independent translations along the time puts the question of Hamiltonian construction possibility rises. Consequences are evident.

- The analog of own reference system, connected with moving particle, will be Schwarzshildean space-time, but not flat Newtonian that.

- All two- and three-dimensional Einsteinian spaces are conformally Euclidean one.

- Cosmological term will certainly appear in the Einsteinian equations, if the corresponding Lagrangian contains the term, corresponding with the volume element.

In 60-70th of the last century unified theory of fundamental interactions was created on a classical level. Its equation system is extended Wheeler-Misner system, describing jointly gravity (by Einsteinian equations) and electromagnetic field in the Riemannian space-time (by Maxvellian equation). If instead of Maxwellian equation will being use Yang-Mills equations this equation system will describe jointly gravity and Yang-Mills field. So, one can combine arbitrary number of fields. Naturally, such system includes in itself the gravity theory of Einstein. Simultaneously it is the gauge field theory, because gauge invariance of each field takes into account in this approach.

Due to its nonlinearity this equation system has nontrivial similar particle solutions. Many solutions of such kind were obtained in 60th for Yang-Mills symmetry group. Some of them have nontrivial space-time topology. But quantization procedure was not applied to them.

\section{Conclusion}

Gauge Field Theory made possible to work out unified picture of all fundamental interactions includind gravity. Therefore distinct scenarios of Universe birth appeared. Standard model of elementary particle interactions is being used for analysis of these processes. Thus cosmic scale phenomena become connected with particle behavior in microworld. This is of course important advance in world cognition. Nature seems more and more various and mysterious. We discover new sides of physical phenomena. Nothing says to us about the end of physics. Psychological crisis in theoretical physics reflects only sharpness of the problems standing before it.

In 1969 "Baldin autumn" stimulated beginning of new direction in the field theory and elementary particle physics. Now we have 45th jubilee of this Seminar. Results are evident. They marked by collection of Nobel prizes. New discoveries are before us. Just strong mathematics can 
be accurate guide in search of truth. Therefore I would like to wish "Badin autumn" was lasting more and more for happiness of Big Science. 